\title{
Theoretical Evaluation of Electroactive Polymer Based Micropump Diaphragm for Air Flow Control
}

\author{
Tian-Bing $\mathrm{Xu}^{\mathrm{a}}, \mathrm{Ji} \mathrm{Su}^{\mathrm{b}}$ and Qiming $\mathrm{Zhang}^{\mathrm{c}}$ \\ a. National Institute of Aerospace, 144 Research Drive, Hampton, VA 23666. \\ b. NASA/Langley Research Center, Hampton, VA 23681 \\ c. Materials Research Institute and Electrical Engineering Department, The Pennsylvania State \\ University, University Park, PA 16802
}

\begin{abstract}
An electroactive polymer (EAP), high energy electron irradiated poly(vinylidene fluoride-trifluoroethylene) [P(VDFTrFE)] copolymer, based actuation micropump diaphragm (PAMPD) have been developed for air flow control. The displacement strokes and profiles as a function of amplifier and frequency of electric field have been characterized. The volume stroke rates (volume rate) as function of electric field, driving frequency have been theoretically evaluated, too. The PAMPD exhibits high volume rate. It is easily tuned with varying of either amplitude or frequency of the applied electric field. In addition, the performance of the diaphragms were modeled and the agreement between the modeling results and experimental data confirms that the response of the diaphragms follow the design parameters. The results demonstrated that the diaphragm can fit some future aerospace applications to replace the traditional complex mechanical systems, increase the control capability and reduce the weight of the future air dynamic control systems.
\end{abstract}

KEYWORDS: Electroactive polymer (EAP), micropump, diaphragm, actuation, displacement, volume rate, pumping speed, clamping ratio.

\section{INTRODUCTION}

Micropumps have been studied for decades and received great attention and interest recently due to the increasing number of applications on a broad range of modern technologies, such as biotechnology, chemical analysis and delivery, and aircraft dynamic control [1]-[3]. A lot of micropumps have been developed using several actuation methods such as the electrostatic [4], piezoelectric [5], thermopneumatic [6], electrochemical [7], shape memory alloy [8], electromagnetic [9], etc. The electrostatic pump [4] is actuated by the electrostatic attraction force. The deflection of the diaphragm can be easily controlled by an applied voltage, and the response time is fast. However, the out pressure and the volume strokes of the electrostatic micropump are small due to the competition between the needed high force level, which favors a small gap between the plates and large displacement range, which requires a large gap. The piezoelectric pump [5] is actuated by the deformation of the piezoelectric material. This actuation method has advantage of large force, high frequency response and simple structure, but the displacement of the device is not high enough due to the intrinsic low strain of piezoelectric ceramics. The thermopneumatic [6] and shape memory alloy pumps [8] exhibiting relatively large flow rates and force levels but the frequency range of the devices is limited due to the requirement of heating and cooling process. The electromagnetic actuation pumps [9] demonstrate high volume stroke and broad frequency range. Unfortunately, the requirement of a wound coil complicates the fabrication process which limits the utility of this type of pump.

Recently, high performance polymer micromachined actuators (PMATs) [10] have been developed based on stretched high energy electron irradiated poly(vinylidene fluoride-trifluoroethylene) [P(VDF-TrFE)] copolymer (HEEIP) [11]-[13] due to the high electromechanical properties of the material. Because of the high electrostrictive strain and high elastic energy density of the HEEIP [11]-[13], the PMAT exhibits a very high stroke level with high load capability, high 
displacement voltage ratio (DVR) and broad frequency response. Although the transverse strain response in the unstretched solution cast high energy-electron irradiated P(VDF-TrFE) copolymer (SCHEEIP) is relatively smaller than the transverse strain in the stretched high energy-electron irradiated P(VDF-TrFE) copolymer along the drawing direction, it is still larger than other electroactive materials [14]. However, there are a few advantages for the unstretched films over the stretched films for device applications. First, the film processing is much simpler for the unstretched films. Second, it can simplify the device process. Third, the strain response is isotropic which is more suitable for circular membrane actuators and is more attractive for use in micro-fluidic devices. In this paper, it will be discussed in detail for the theoretical design and operation principle, fabrication and characterization, modeling, and performance of a high energy electron irradiated poly(vinylidene fluoride-trifluoroethylene) [P(VDF-TrFE)] copolymer based circular actuation micropump diaphragm (PAMPD).
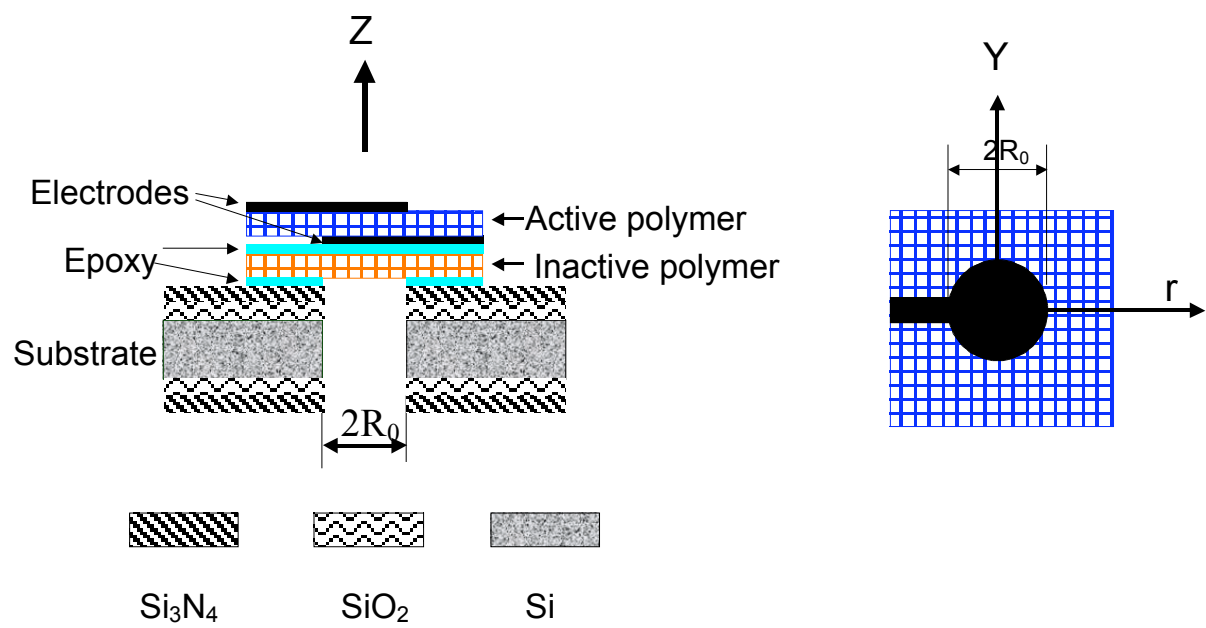

Fig. 1. Configuration of the micropump diaphragm actuator based on the solution cast and high-energy electron irradiated P(VDF-TrFE) copolymer (SCHEEIP) on a silicon bulk micromachined substrate. (a) The cross section view of the device, and (b) the top view of the active layer.

\section{DESIGN AND OPERATION PRINCIPLE OF PAMPD}

\subsection{Design of the polymer based actuation micropump diaphragm (PAMPD)}

Utilizing relatively large, positive isotropic transverse electrostrictive strain with high elastic modulus in both of $\mathrm{S}_{1}$ and $\mathrm{S}_{2}$, [12] of the solution cast and high-energy electron irradiated P(VDF-TrFE) copolymer (SCHEEIP); a circular polymer micropump diaphragm actuator configuration adopted for this investigation is shown schematically in Fig. 1. The active polymer used here was characterized for its electromechanical response, and under a field of $100 \mathrm{~V} / \mu \mathrm{m}$, a transverse strain $\left(S_{l}\right)$ of $0.8 \%$ was observed. The elastic modulus was $0.4 \mathrm{GPa}$. The active polymer, which is a film $(\sim 10 \square \mathrm{m}$ thickness) of SCHEEIP with sputtered gold electrodes (Fig. 1(b)), was bonded to an inactive polymer film using Spurr epoxy (Polysciences, Inc., Warrington, PA) to form a unimorph actuator. In the current design, the inactive polymer is the same as the active polymer without electrodes for the first time investigation. The thickness ratio and elastic constant ratio between the two polymer layers can be varied to tailor the actuators for different applications. This inactive layer will add mechanical stress as the electrostrictive SCHEEIP is electrically activated. This stress will constrain the active element, reduce the strain response, and control the motion of the device to a desired direction for this application. Under an electric field, the expansion of the active polymer generates an upward motion of the unimorph actuator (the zdirection in Fig. 1). The edge of the circular diaphragm actuator was fixed on a bulk Si micromachined substrate to combine with an integrated electronic circuit and define the diameter of the actuator. The diameter of the PAMPD invested in this paper is $2 R_{0}=1 \mathrm{~mm}$. 


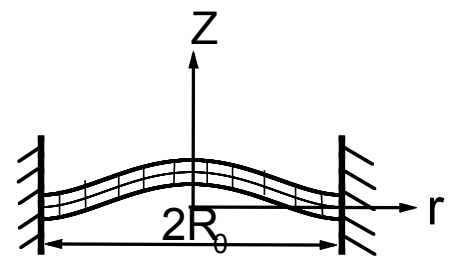

Fig. 2. Configuration of the motion of the PAMPDs under an electric filed which is modeled based on a circular plate with clamped edges under uniform mechanical pressure in the lateral direction

\subsection{PAMPD Operation principle}

The device was modeled as a circular plate with clamped edges under uniform mechanical pressure in the lateral direction $(z)$. Since the thickness $t$ of the device was much smaller than the diameter $\left(2 \mathrm{R}_{0}\right)$ of the device, we assume that the active layer thickness of the device will not change during operation. When an electric field is applied to the device, the active layer will expand along the film direction. Meanwhile, the inactive layer constrains the motion of the active layer. This interaction will result in a uniform load distribution on the circular diaphragm with the edge clamped. This actuation process is schematically shown in Fig. 2 and the displacement along the r-direction can be expressed as, [15]

$$
z=c\left[\left(R_{0}\right)^{2} \square r^{2}\right]
$$

where $c$ is a constant which is determined by effective transverse strain $\mathrm{S}_{\mathrm{e}}$ of the film, i.e.,

$$
\prod_{\square R_{0}}^{R_{0}} d r d z
$$

which is based on the geometric constraint, that should be equal to the length of polymer film in the diameter after the strain $\mathrm{S}_{\mathrm{e}}$ that the total length, in a radial direction, of the actuator after the actuation. Because of the inactive polymer layer, $\mathrm{S}_{\mathrm{e}}$ is different from the strain $\mathrm{S}_{1}$ of the active polymer measured in the free condition. From the force balance condition in the r-direction, $\mathrm{S}_{\mathrm{e}}$ is related to $\mathrm{S}_{1}$ as,

$$
E_{a} t_{a}\left(S_{1} \square S_{e}\right)=E_{n} t_{n} S_{n}
$$

which leads to $\mathrm{S}_{\mathrm{e}}=S_{1} /(1+k)$, where $\mathrm{k}=\left(E_{n} t_{n}\right) /\left(E_{a} t_{a}\right)$ is a measure of the clamping effect due to inactive layer (clamping ratio[10]). To include the contribution from the metal electrodes and Spurr epoxy bonding layer, k should be modified to $\mathrm{k}=\left(\square E_{n i} t_{n i}\right) /\left(E_{a} t_{a}\right)$, where $\mathrm{E}_{\mathrm{ni}}$ and $\mathrm{t}_{\mathrm{ni}}$ are the elastic modulus and thickness of $\mathrm{i}$-th inactive layer, respectively. It should be noted that in the derivation, we omitted the mechanical clamping effect at the edge of the circular diaphragm $\left(\mathrm{r}= \pm R_{0}\right)$ which will reduce $S_{l}$ even if $\mathrm{k}=0$. All the analysis is based on the assumption that $\mathrm{R}_{0}>>\mathrm{t}$, where $\mathrm{t}$ is the total thickness of the diaphragm actuator.

The volume change for the micropump diaphragm from flat $(\mathrm{z}=0)$ to a certain degree of the deformation $\left(\mathrm{z}=\mathrm{Z}_{0}\right.$ at the center) can be expressed as

$$
V=\underset{0}{z_{0}} \square r^{2} d z
$$

From equation (1) we can obtain

$$
d z=\square 4 c\left(R_{0}^{2} \square r^{2}\right)^{2} r d r
$$

In the meanwhile, $r$ equal $R_{0}$, and 0 when $z$ equal 0 and $z_{0}$, respectively. Then, the volume change can be written as

$$
V=\underset{0}{R_{0}} 4 \square c r^{3}\left[R_{0}^{2} \square r^{2}\right] r=\frac{1}{3} c R_{0}^{6}
$$

Therefore, the volume rate (pumping speed at 1 atmosphere pressure) of the diaphragm can be expressed as

$$
V_{s}=2 \square 60 \square f_{p} V=40 c f_{p} R_{0}^{6}
$$


where, 60 is the convert constant from the unit of volume change per second to the unit of volume change per minute, which is expressed as $\square \mathrm{L} / \mathrm{min}$ in the fields of micropump research; $f_{p}$ is the frequency of the pumping motion of the micropump diaphragm. For the electrostrictive material based active diaphragm, the frequency of the diaphragm will be twice that of the frequency of the driving electric field [12], i.e.,

$$
f_{p}=2 f_{e}
$$

where, $f_{e}$ is the frequency of the driving electric field.
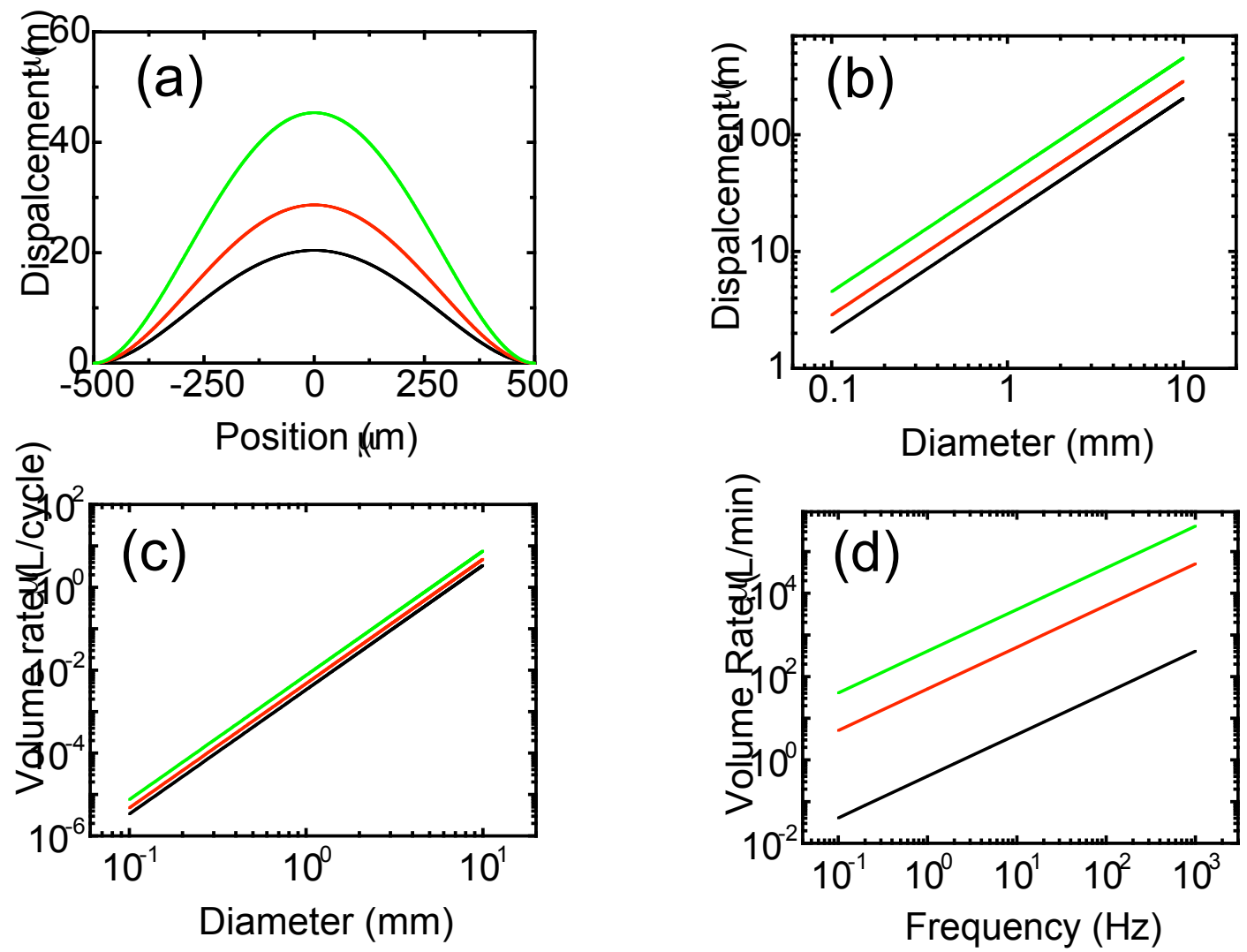

Fig. 3 The baseline theoretical modeled the results of the PAMPDs. (a) The displacement as a function of position along a diameter. From bottom to up for the effective strain of $0.1 \%, 0.2 \%$, and $0.5 \%$, respectively. (b) The maximum displacement as a function of diameter $\left(2 \mathrm{R}_{0}\right)$, from bottom to up for effective strain of $0.1 \%, 0.2 \%$, and $0.5 \%$, respectively. (c) Volume rate (volume change per cycle) as a function of diameter $\left(2 \mathrm{R}_{0}\right)$, from bottom to up for effective strain of $0.1 \%, 0.2 \%$, and $0.5 \%$, respectively. (d) Volume rate as a function of frequency at effective strain of $0.1 \%$, from bottom to up for diameter of $1 \mathrm{~mm}, 5 \mathrm{~mm}$ and $10 \mathrm{~mm}$, respectively.

Using the above equations, the modeling results of the PAMPD are presented in Fig. 3. Fig. 3 (a) presents the displacement as a function of position along a diameter of the diaphragm at effective strain $S_{e}=0.1 \%, 0.2 \%$ and $0.5 \%$ respectively. The maximum displacements, at the center of the diaphragm, can reach 20_m, 28_m, and 45_m for $S_{e}=$ $0.1 \%, 0.2 \%$ and $0.5 \%$ respectively. Fig. 3 (b) exhibits the maximum displacement as a function of the diameter of the PAMPDs for effective strain of $0.1 \%, 0.2 \%$, and $0.5 \%$, respectively. There is a linear relation between the maximum displacement and the diameter of the diaphragm for an effective strain $S_{e}$. Fig. 3 (c) shows the volume stroke rate (volume rate, volume change per cycle) as a function of diameter of a PAMPD. The result indicates that the volume rate is square of the diameter of the PAMPD. Fig. 3 (d) predicts the volume rate as a function of frequency at effective strain 
of $0.1 \%$, for diameter of $1 \mathrm{~mm}, 5 \mathrm{~mm}$ and $10 \mathrm{~mm}$, respectively. It indicates that the volume rate is linearly increases with the frequency increase when the effective strain keeping constant for a PAMPD.

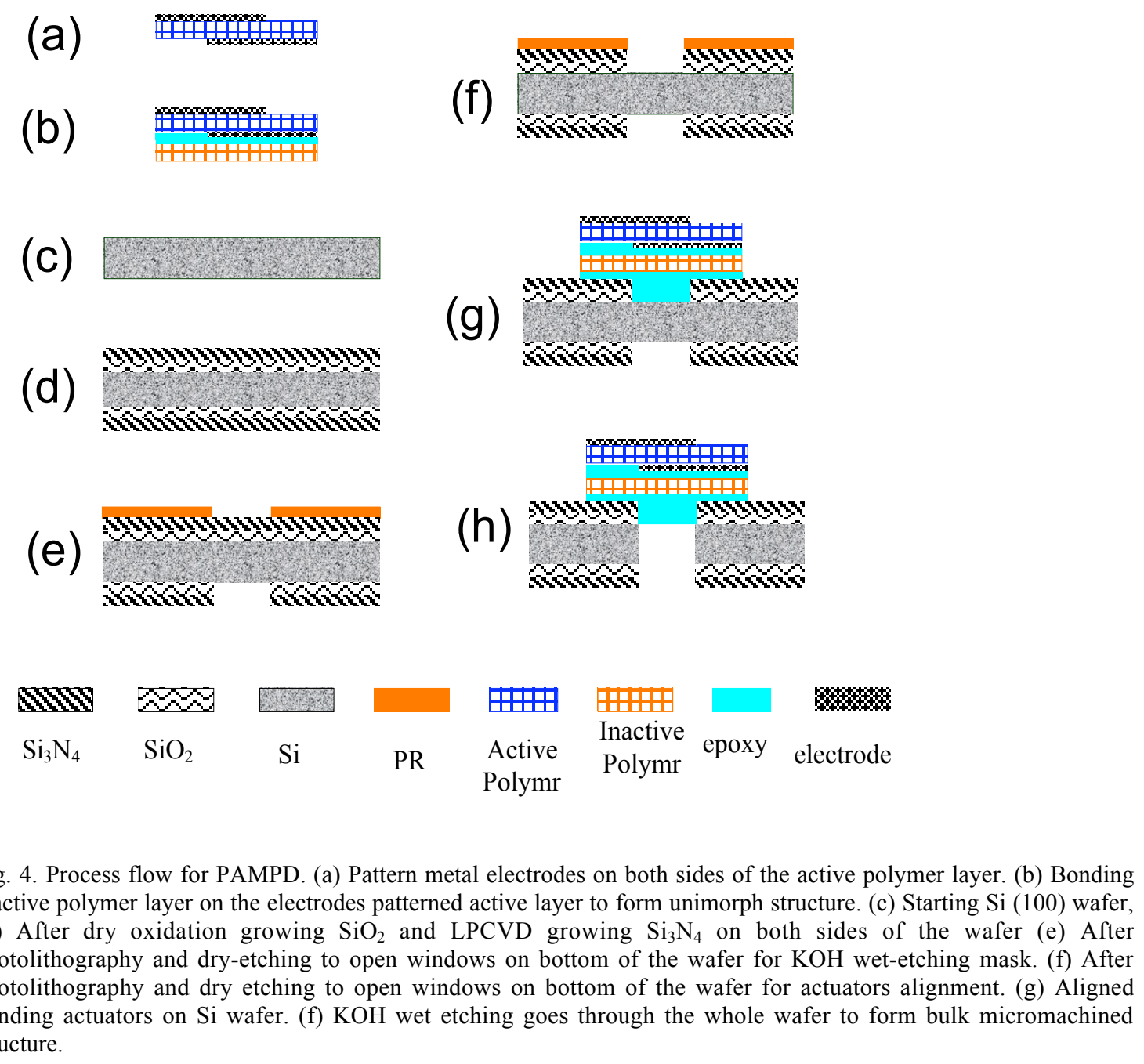

\section{DEVICE FABRICATION AND CHARACTERIZATION}

The process detail of the unstretched high energy-electron irradiated $\mathrm{P}(\mathrm{VDF}-\mathrm{TrFE})$ copolymer film, EAP film, was discussed in reference [12]. The $100 \mathrm{~nm}$ gold electrodes were patterned by sputtering on both side of the active layer as shown in Fig. 4 (a) utilizing shadow mask. The inactive polymer layer was bonded shown in Fig. 4 (b) with Spurr epoxy (Polysciences, Inc., Warrington, PA) cured at room temperature for 1 week. The bulk micro-machined silicon substrate was fabricated at the Penn State Nanofabrication Facility using standard bulk silicon micromachining technology utilizing the $\mathrm{KOH}$ anisotropic wet-etching technique [16]. A 400Øm thick 4" in diameter Si (100) wafer was selected as a substrate as shown in Fig. 4 (c). A $250 \mathrm{~nm} \mathrm{Si}_{3} \mathrm{~N}_{4}$ film was selected as a wet-etching mask layer due to the larger expected etching ratio between $\mathrm{Si}$ and $\mathrm{Si}_{3} \mathrm{~N}_{4}$. To eliminate the stress between $\mathrm{Si}$ and $\mathrm{Si}_{3} \mathrm{~N}_{4}$ [17], a 100nm $\mathrm{SiO}_{2}$ layer was selected as a buried layer. $\mathrm{The} \mathrm{SiO}_{2}$ layer was grown using the dry oxidation method at $950{ }^{\circ} \mathrm{C}$ on both sides of the wafer, and the $\mathrm{Si}_{3} \mathrm{~N}_{4}$ layer was grown using the LPCVD method on both sides of the wafer as shown in Fig. 4 (d). Photoresist was then applied and patterned with a photomask utilizing photolithography technology. As the wet-etching mask, the windows of $\mathrm{Si}_{3} \mathrm{~N}_{4}$ as well as $\mathrm{SiO}_{2}$, were opened using standard RIE dry etching technology with the photoresist as the dry-etching 
mask shown in Fig. 4 (e). In order to align between the patterned electrodes and the wet-etching window, and reduce the thermal budget on the polymer actuators, the top $\mathrm{Si}_{3} \mathrm{~N}_{4}$ as well as $\mathrm{SiO}_{2}$ layer windows were opened utilizing photolithography and dry etching technology with the same mask shown in Fig. 4 (f). Afterward, the polymer unimorph actuator was bonded onto the Si substrate utilizing epoxy at room temperature. Finally, the $\mathrm{KOH}$ wet-etching process was carried out at $70{ }^{\circ} \mathrm{C}$ for $10 \mathrm{hrs}$ going through the whole wafer to form the bulk micromachined device shown in Fig. $4(\mathrm{~h})$.

The displacement response was characterized by a laser vibrometer (Polytec PI, Inc, model OFV-512) which has a beam size of $5 \square \mathrm{m}$ and upper measuring frequency of $1.5 \mathrm{MHz}$ as shown in Fig. 5. In order to reduce the measurement noises, the setup is built on an air isolated optical table. The measured device was fixed on a 3- dimensional stages based sample bed. The laser vibrometer head was hooded on a sensor head holder which is based on an x-y-z 3-dimensional with two additional axes rotation to adjust the laser beam to focus and perpendicular to the measured surface. The driving voltage of the device is a locking amplifier output signal amplified by a Trek Amplifier (Trek. 610D). The detected displacement signal output from the vibrometer controller to an oscilloscope to get a waveform signal, and to the lock-in amplifier to convert the analog signal to a digital signal.

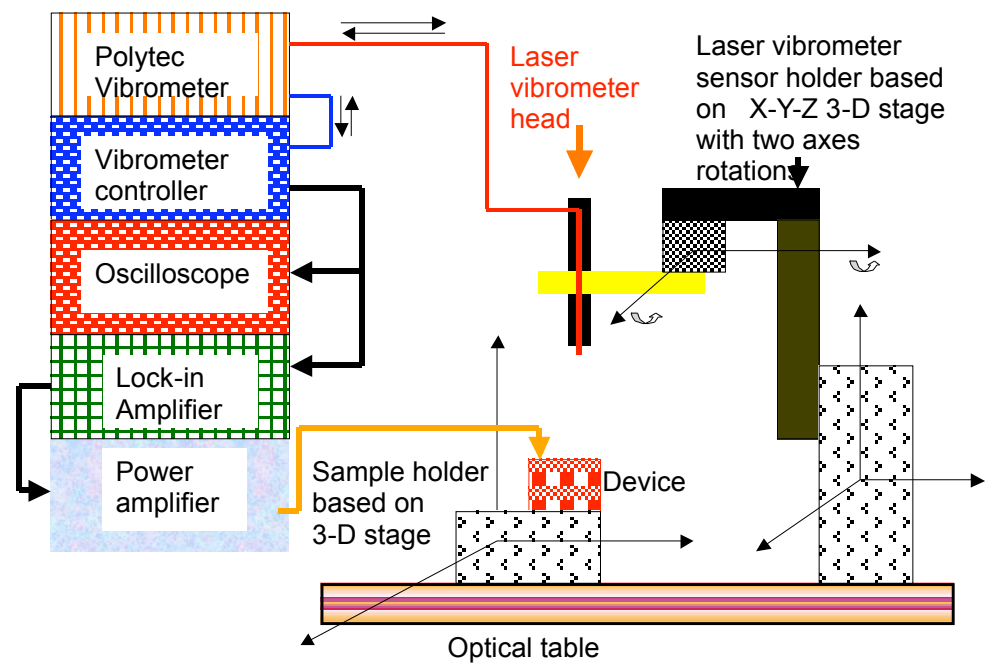

Fig. 5. Diagram of the displacement measurement setup for the PAMPD.

\section{DEVICE PERFORMANCE}

In order to evaluate the performance of the PAMPD, we first present in Fig. 6 a displacement profiles measured on a PAMPD as a function of $\mathrm{r}$ along an arbitrary diameter. The applied AC electric field is $80 \mathrm{~V} / \mathrm{m}$ at frequency of $20 \mathrm{~Hz}$. The maximum displacement at the center of the PAMPD reaches to $18 \mu \mathrm{m}$. The solid line is fitted from equations (1) (2) and (3) which match the experimental results (dots) quite well. This indicates that the actuation function as anticipated. The effective transverse strain, modeled from the displacement profile, is around $0.09 \%$. The clamping ratio of the inactive portion is $\mathrm{k}=5$. This is reasonable considering the fact that in addition to the inactive layer $(\mathrm{k}=1)$, there are Spurr epoxy layers (the elastic modulus $\mathrm{G}=5 \mathrm{GPa}$ and thickness $\sim 1 \square \mathrm{m}$ ) [10], [13], Au electrodes (200 $\mathrm{nm}$ total thickness and elastic modulus $\sim 100 \mathrm{GPa}$ ), and the clamp effect at the fixed edge of the circular diaphragm. By reducing $\mathrm{k}$ to equal 1 , in the future design, the displacement output can be significantly increased from the prediction of Fig. 3. We point out that 
in the current PAMPD design: only the top polymer film is active, resulting in an upward motion of the actuator. If needed, one can alternatively drive the two polymer layers, which will result in the motion of the actuator in both upward and downward directions to double the displacement output for air control.

The displacement at the center of the PAMPD versus applied electric field was measured and the result is presented in Fig. 7. The maximum displacement is more than 21_m when the applied AC electric field at $100 \mathrm{~V} / \mathrm{m}$ with frequency of $10 \mathrm{~Hz}$. It can compete with the displacements of most micropumps at the diameter of around $5 \mathrm{~mm}$ [3].

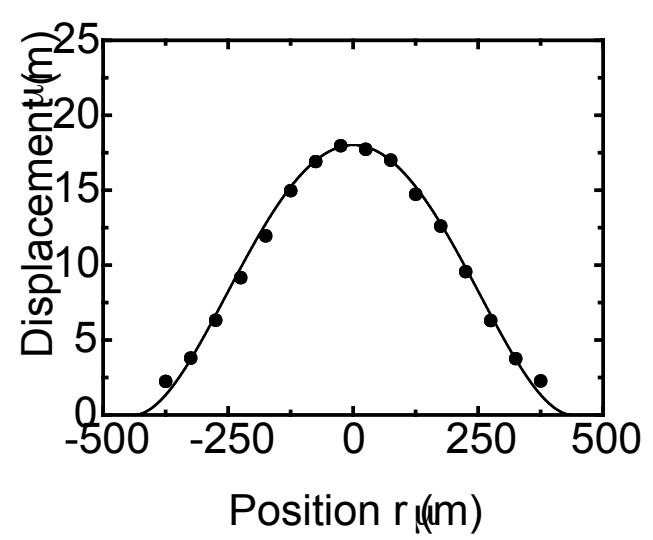

FIG. 6. Displacement profile for a PAMPD, circular dots (measured at $20 \mathrm{~Hz}$ and amplitude of $80 \mathrm{~V} / \square \mathrm{m}$ field), along the diameter-direction where the dots are measured data and solid curves are fitting from equation (1).

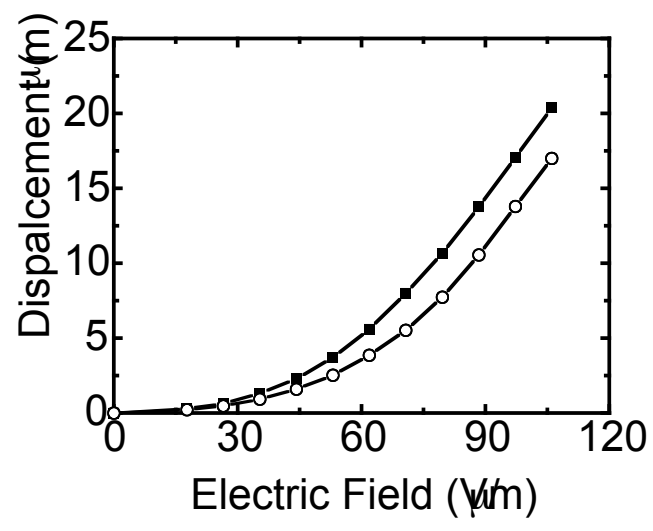

FIG. 7. (a) Displacement at the center of a PAMPD as a function of the applied electric AC field. The square solid dots is for the driving frequency of 10 $\mathrm{Hz}$, the circular open dots are the driving frequency at $100 \mathrm{~Hz}$, and the lines are drawn to guide eyes.

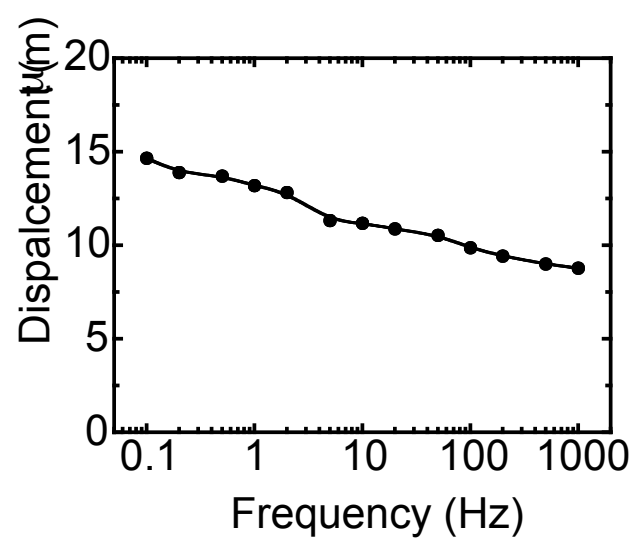

FIG. 8. Displacement as a function of frequency for the PAMPD measured at AC electric field of $60 \mathrm{~V} / \mu \mathrm{m}$. The solid circles are the measured data, and the dash line is drawn to guide eyes. 
In order to demonstrate the high frequency performance of the PAMPD, the displacements as a function of frequency for PAMPD at the fixed amplitude of electric field as $60 \mathrm{~V} / \mu \mathrm{m}$ is presented in Fig 8 . The maximum frequency of the measurement was limited by the frequency response of the Trek 610D amplifier. The dispersion of the displacement with frequency increasing is less than $30 \%$ over 4 frequency decades. The results demonstrate two main features of the PAMPD investigated: (a) it possesses a high displacement, and (b) it is capable of operating over a broad frequency range. As we reported in reference [18], the electromechanical coupling factor of the materials remains unchanged at high frequencies; it is clear that the PAMPD can work as broadband frequency and operated at no resonance condition.

In order to evaluate the pumping capability of the active micropump diaphragm, the calculated volume rate (pumping speed for air control when the air pressure close to 1 atmosphere pressure) as a function of electric field based on the experimental data in Fig. 7 and the equations (7) and (8) is presented in Fig. 9. The volume rate is increasing with the electric field increase almost follow: $V_{s}=E^{2.5}, \mathrm{E}$ is the electric field. The volume rate is over $30 \_\mathrm{L} / \mathrm{min}$ at $100 \mathrm{~V} / \mathrm{m}$ with frequency of $100 \mathrm{~Hz}$. It is higher than most piezoelectric pump at diameter over $5 \mathrm{~mm}$. If keep the same effective strain level and increase the diameter of the PAMPD to $5 \mathrm{~mm}$ the volume rate will be more than $5000 \mathrm{~L} / \mathrm{min}$ at frequency of $100 \mathrm{~Hz}$ from the prediction of Fig 3 (d). It will several tens time higher than the same size piezoelectric micropump reported recently [3]. The results are also indicating that the volume rate is very sensitive to the amplitude of the applied electric field as well as the frequency of the electric field. The pumping speed of the PAMPD micropump may be smaller than the measured and calculated volume rate. However, it should not reduce too much at close to the pressure of 1 atmosphere for air dynamic control, due to the high load capability of this kind actuator from our previous report in reference [10].

The calculated Volume rate (pumping speed) as a function of frequency for the pump diaphragm at electric field of $60 \mathrm{~V} / \square \mathrm{m}$ based on the data of figure 6 and equations of (7) and (8) is presented in Fig. 10. The volume rate is 176_L/min at $1000 \mathrm{~Hz}$. The high performance is due to the high transverse strain and high frequency response of the electroactive polymer. The volume rate is very sensitive to the frequency at same amplitude of the applied electric field. A PAMPD based air dynamic flow controller can be easily tuned by varying the frequency of the electric field.
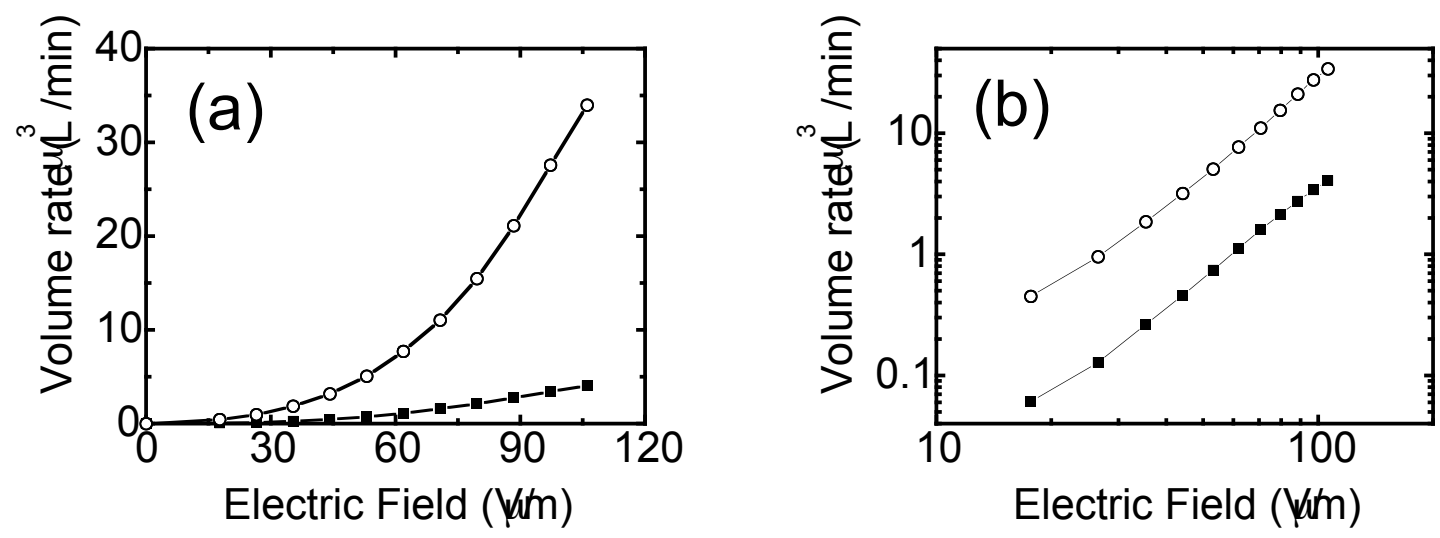

FIG. 9. The calculated volume rate (pumping speed) as a function of electric field based on the data of Fig. 7 and equations (7) and (8). The solid square dots are frequency at $10 \mathrm{~Hz}$ and open circular dots are frequency at $100 \mathrm{~Hz}$. The solid lines are drawn to guide eyes. (a) is the linear plot and (b) is the log plot. 


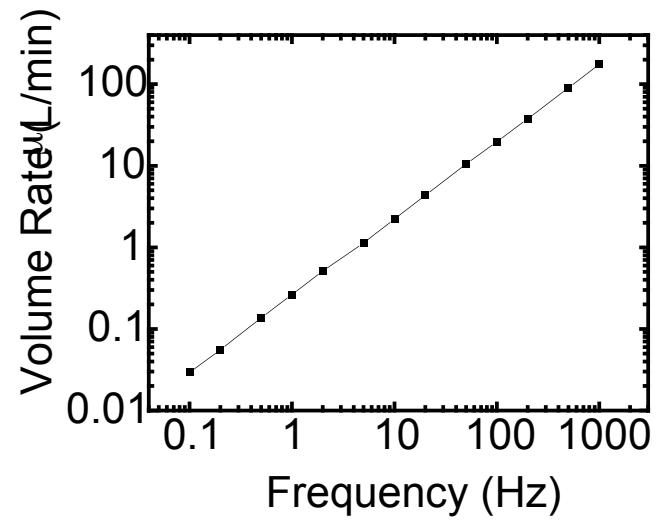

FIG. 10. The calculated Volume rate (pumping speed) as a function of frequency for the pump diaphragm at electric field of 60V/ $/ \mathrm{m}$ based on the data of Fig. 8 and equations of (7) and (8). The solid line is drawn to guide eyes.

\section{CONCLUDING REMARKS}

In summary, PAMPD based on EAP was designed, modeled, fabricated and characterized. The displacement at the center of the PAMPD can reach $21 \_\mathrm{m}$ at $100 \mathrm{~V} / \mathrm{m}$ with driving frequency of $10 \mathrm{~Hz}$. The dispersion of the displacement under fixed amplitude of electric field was also characterized and the dispersion is less than $30 \%$ for more than 4 frequency decades $(1 \mathrm{~Hz}$ to $1000 \mathrm{~Hz})$. The performance of the PAMPD has been theoretically modeled in terms of the properties of the EAP copolymer and the geometry of the device. The experimental results of the device responses matched quite well with the model prediction. The volume rate of the PAMPD was theoretically evaluated based on the displacement profile at different driving electric field conditions. It is over $30 \mathrm{~L} / \mathrm{min}$ at $100 \mathrm{~V} / \mathrm{m}$ with frequency of $100 \mathrm{~Hz}$ and $176 \_\mathrm{L} / \mathrm{min}$ at $1000 \mathrm{~Hz}, 60 \mathrm{~V} / \mathrm{m}$. The volume rate of the PAMPD is several tens time large than the most piezoelectric ceramic based micropump diaphragm at same size for current reported results. The volume rates are very sensitive to bath the amplitude and the frequency of the applied electric field. In the meanwhile, the volume rate is very easy tuned by either the amplitude or the frequency of the applied electric field. The performances of the PAMPD demonstrated here indicate that this type of electrostrictive P(VDF-TrFE)-based MEMS devices are very attractive for micro-pumps, and air dynamic flow controls.

The authors wish to thank Dr. Joycelyn Harrison for discussion concerning this work. This work was supported by NIH, DARPA and NASA. 


\section{REFERENCES}

1. L. Lofdahl, and M. Gad-el-Hak, "MEMS applications in turbulence and flow control," Progress in Aerospace Sciences Vol. 35, pp. 101-203, 1999.

2. V. K. Varadan and V. V. Varadan, "Microsensors, microelectromechanical systems (MEMS), and electronics for smart structures and systems," Smart Mater. Struct. Vol. 9, pp. 953-972, 2000.

3. N.-T. Nguyen, X. Huang, and T. K. Chuan, "MEMS-Micropumps: A Review," Journal of Fluids Engineering Transaction of the ASME., Vol. 124, pp. 384-392, 2002.

4. O. Francais, and I. Dufour, "Enhancement of elementary displaced volume with electrostatically actuated diaphragms: Application to electrostatic micropumps," J. Micromech. Microeng. Vol. 10 (2), pp. 281-286, 2000.

5. Y. H. Mu, N.P. Hung, and K.A. Ngoi, "Optimisation design of a piezoelectric micropump," Intern. J. Adv. Manuf. Technol. Vol. 15, pp. 573-576, 1999.

6. A. Wego, H.-W. Glock, L. Pagel, and S. Richter, "Investigations on thermopneumatic volume actuators based on PCB technology," Sensors \& Actuators, Vol. 93, pp. 95-102, 2001.

7. C. R. Neagu, J.G.E. Gardeniers, M. Elwenspoek, and J.J. Kelly, "An electrochemical microactuator: principle and first results, J. Microelectromech. Sys. Vol. 5 (1), pp. 2-9, 1996.

8. W. L. Benard, H. Kahn, A. H. Heuer, and M. A. Huff, "Thin-film shape-memory alloy actuated micropumps," J. Microelectromech. Syst. Vol. 7 (2), pp. 245-251 1998.

9. J. Joung, J. Shen, and P. Grodzinski, "Micropumps Based on Alternating High-Granient Magnetic fields," IEEE Trans. Magn, Vol. 36, no. 4, pp. 2012-4014, 2000.

10. T.-B. $\mathrm{Xu}_{2} \mathrm{Z}$.-Y. Cheng, and Q. M. Zhang, "High-performance micromachined unimorph actuators based on electrostrictive poly(vinylidede fluoride-trifluoroethylene) copolymer” Appl. Phys. Lett. Vol. 80, (6), pp. 1082-1084, 2002.

11. Q. M. Zhang, V. Bharti, and X. Zhao, "Giant electrostriction and relaxor ferroelectric behavior in electronirradiated poly(vinylidene fluoride-trifluoroethylene) copolymer", Science, Vol. 280, pp.2101-2104, 1998.

12. Z. -Y. Cheng, T. -B. Xu, V. Bharti, S. Wang, and Q. M. Zhang, "Transverse strain responses in the electrostrictive poly(vinylidene fluoride-trifluoroethylene) copolymer”, Appl. Phys. Lett. Vol. 74, pp. 19011903, 1999.

13. Z.-Y. Cheng, T.-B. Xu, Q. M. Zhang, R. Meyer, J. D. Van Tol, and J. Hughes, "Design, fabrication and performance of a flextesional transducer based on electrostrictive polyvinylidene fluoride-trifluoroethylene copolymer," IEEE Trans. Ultrason., Ferroelect., Freq. Contr. Vol. 49 (9), pp. 1312-1320, 2002.

14. T.-B. Xu, "Development of electromechanical devices based on newly developed electroactive P(VDFTrFE) polymer," Ph.D. Thesis, The Pennsylvania State University 2002.

15. S. Timoshenko, and S. Woinowsky-Krieger, Theory of plates and shells, Second Edition, McGRAWHILL,Inc., New York, 1959.

16. M. Madou, Fundamentals of Microfabrication, CRC Press, New York 1997.

17. J.-H. Mo, A. L. Robinson, D. E. Fitting, F. L. Terry, P. L. Carson, "Micromachining for improvement of integrated ultrasonic transducer sensitivity," IEEE Trans. Electron Dev. Vol. 37 (1), pp. 134-140 1990.

18. T.-B. Xu, Z.-Y. Cheng, T. Mai, Yu Lu, and Q.M. Zhang, "Electromechanical coupling factor of electrostrictive P(VDF-TrFE) copolymer," 2000 IEEE Ultrasonics Symposium Proc. Vol. 2, pp. 997-1000, 2000. 